
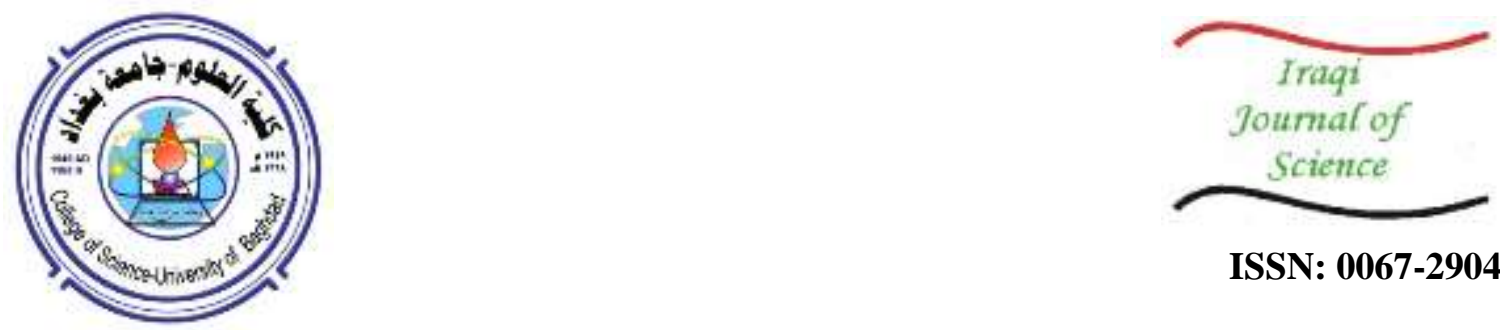

ISSN: 0067-2904

\title{
Generalized Strong Commutativity Preserving Centralizers of Semiprime $\Gamma$ - Rings
}

\author{
Shahed Ali Hamil", Abdulrahman H. Majeed \\ Department of Mathematics, College of Science, University of Baghdad, Baghdad, Iraq
}

\begin{abstract}
In this paper, we introduce the concept of generalized strong commutativity (Cocommutativity) preserving right centralizers on a subset of a $\Gamma$-ring. And we generalize some results of a classical ring to a gamma ring.
\end{abstract}

Keywords: $\Gamma$-ring, semiprime $\Gamma$-ring, generalized strong commutativity preserving right centralizer, generalized strong Cocommutativity preserving right centralizer.
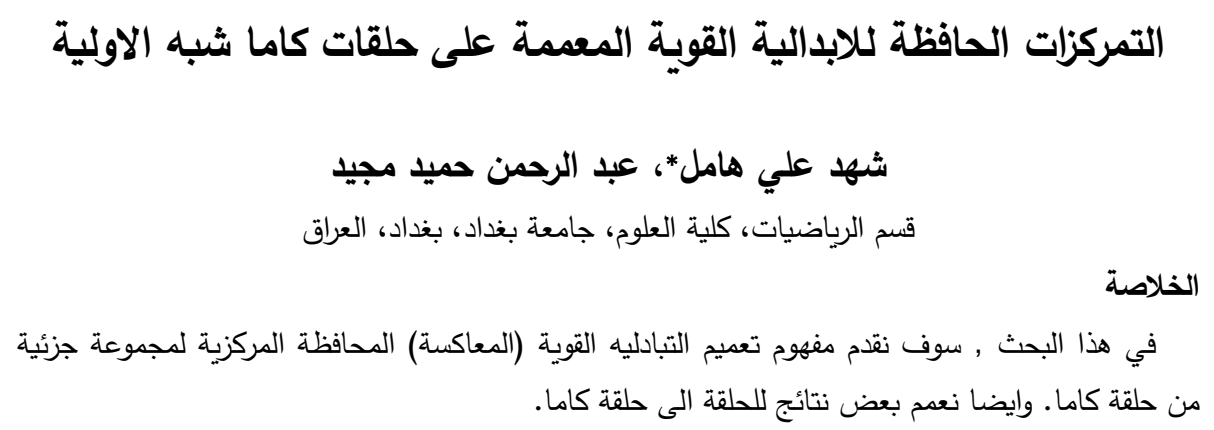

\section{Introduction}

Let $M$ and $\Gamma$ be additive abelian groups. If there exists a mapping of $M \times \Gamma \times M \rightarrow M,(a, \alpha, b)$ $\rightarrow a \alpha b$ which satisfies the conditions

(i) $a \alpha b \in M$ (ii) $(a+b) \alpha c=a \alpha c+b \alpha c, a(\alpha+\beta) c=a \alpha c+a \beta c, a \alpha(b+c)=a \alpha b+a \alpha c$ (iii) $(a \alpha b) \beta c=$ $a \alpha(b \beta c)$ for all $a, b, c \in M$ and $\alpha, \beta \in \Gamma$, then $M$ is called a $\Gamma$-ring. Every $\operatorname{ring} M$ is a $\Gamma$-ring with $M=$ $\Gamma$. However a $\Gamma$-ring need not be a ring. Gamma rings, more general than rings, were introduced by Nobusawa [1]. Bernes [2] weakened slightly the conditions in the definition of $\Gamma$-ring. Let $M$ be a $\Gamma$ ring. Then an additive subgroup $N$ of $M$ is called a left (right) ideal of $M$ if $M \Gamma N \subset N(N \Gamma M \subset N)$. If $N$ is both a left and a right ideal, then we say $N$ is an ideal of $M$. Suppose again that $M$ is a $\Gamma$-ring. Then $M$ is said to be a 2-torsion free if $2 a=0$ implies $a=0$ for all $a \in M$. A $\Gamma$-ring $M$ is said to be prime if $a \Gamma М Г b=(0)$ with $a, b \in M$, implies $a=0$ or $b=0$ and semiprime if $a \Gamma M \Gamma a=(0)$ with $a \in M$ implies $a$ $=0$. Furthermore, $M$ is said to be commutative $\Gamma$-ring if $a \alpha b=b \alpha a$ for all $a, b \in M$ and $\alpha \in \Gamma$. Moreover, the set $Z(M)=\{a \in M: a \alpha b=b \alpha a$ for all $\alpha \in \Gamma, b \in M\}$ is called the center of the $\Gamma$-ring $M$. If $M$ is a $\Gamma$-ring, then $[a, b]_{\alpha}=a \alpha b-b \alpha a$ is known as the commutator of $a$ and $b$ with respect to $\alpha$, where $a, b \in M$ and $\alpha \in \Gamma$ and $(a o b)_{\alpha}=a \alpha b+b \alpha a$ is known as an anticommutator of $a$ and $b$ with respect to $\alpha$. We make the basic commutator identities:

$[a \alpha b, c]_{\beta}=[a, c]_{\beta} \alpha b+a[\alpha, \beta]_{\mathrm{c}} b+a \alpha[b, c]_{\beta}$ and $[a, b \alpha c]_{\beta}=[a, b]_{\beta} \alpha c+b[\alpha, \beta] a c+b \alpha[a, c]_{\beta}$, for all $a$, b. $c \in M$ and $\alpha, \beta \in \Gamma$. We consider the following assumption: $a \alpha b \beta c=a \beta b \alpha c$, for all $a, b, c \in M$, and $\alpha, \beta \in \Gamma$.

According to the assumption $(A)$, the above two identities reduce to

$[a \alpha b, c]_{\beta}=[a, c]_{\beta} \alpha b+a \alpha[b, c]_{\beta}$ and $[a, b \alpha c]_{\beta}=[a, b]_{\beta} \alpha c+b \alpha[a, c]_{\beta}$, which we extensively used. An additive mapping $T: M \rightarrow M$ is a left(right) centralizer if $T(a \alpha b)=$ $T(a) \alpha b(T(a \alpha b)=a \alpha T(b))$ holds for all $a, b \in M$ and $\alpha \in \Gamma$. A centralizer is an additive mapping which 
is both a left and a right centralizer. We shall restrict our attention on left centralizer, since all results of right centralizers are the same as left centralizers. An additive mapping $D: M \rightarrow M$ is called a derivation if $D(a \alpha b)=D(a) \alpha b+a \alpha D(b)$ holds for all $a, b \in M$, and $\alpha \in \Gamma$ and is called a Jordan derivation if $D(a \alpha a)=D(a) \alpha a+a \alpha D(a)$ for all $a \in M$ and $\alpha \in \Gamma$. An additive mapping $T: M \rightarrow M$ is Jordan left(right) centralizer if $T(a \alpha a)=T(a) \alpha a(T(a \alpha a)=a \alpha T(a))$ for all $a \in M$, and $\alpha \in \Gamma$. Every left centralizer is a Jordan left centralizer but the converse is not true in general. An additive mappings $T$ : $M \rightarrow M$ is called a Jordan centralizer if $T(a \alpha b+b \alpha a)=T(a) \alpha b+b \alpha T(a)$, for all $a, b \in M$ and $\alpha \in \Gamma$. Every centralizer is a Jordan centralizer but Jordan centralizer is not in general a centralizer. Bernes [2], Kyuno [3] and Luh [4] studied the structure of $\Gamma$-rings and obtained various generalizations of corresponding parts in ring theory. Borut Zalar [5] worked on centralizers of semiprime rings and prove that Jordan centralizers and centralizers of this rings coincide. Joso Vukman [6-8] developed some remarkable results using centralizers on prime and semiprime rings. In [9], Hoque and Paul proved that every Jordan centralizer of a 2.torsion free semiprime $\Gamma$-ring satisfying a certain assumption is a centralizer. Also, they proved in [10], if $T$ is an additive mapping on a 2.torsion free semiprime $\Gamma$-ring $M$ with a certain assumption such that $T(a \alpha b \beta a)=a \alpha T(b) \beta a$, for all $a, b \in M$ and $\alpha$, $\beta \in \Gamma$, then $T$ is a centralizer. And in [11], if $2 T(a \alpha b \beta a)=T(a) \alpha b \beta a+a \alpha b \beta T(a)$, for all $a, b \in M$ and $\alpha, \beta \in \Gamma$, then $T$ is also a centralizer.

In this paper, we generalize some results of Shaker [12] and Abduljaleel [13] in gamma rings,

To prove our main results, we need the following lammas.

Lemma 1.1[9] Le t $M$ be a semiprime $\Gamma$-ring. If $a, b \in M$ and $\alpha, \beta \in \Gamma$ are such that $a \alpha x \beta b=0$ for all $x \in M$, then $a \alpha b=b \alpha a=0$.

Lemma 1.2 [9]Let $M$ be a semiprime $\Gamma$-ring and $A: M \times M \rightarrow M$ biadditive mapping. If $A(a, b) \alpha w \beta B(a, b)=0$ for all $a, b, w \in M$ and $\alpha, \beta \in \Gamma$, then $A(a, b) \alpha w \beta B(u, v)=0$ for all $a, b, u, v \in M$ and $\alpha, \beta \in \Gamma$.

Lemma 1.3[9] Let $M$ be a semiprime $\Gamma$-ring satisfying the assumption (A) and $a \in M$ be some fixed element. If $a \alpha[x, y] \beta=0$ for all $a, b \in M$ and $\alpha, \beta \in \Gamma$, then there exists an ideal $U$ of $M$ such that $a \in$ $U \subset Z(M)$ holds.

\section{Generalized strong commutativity preserving centralizers on semiprime $\Gamma$-ring.}

We will introduce the following definition.

Definition 2.1 . Let $N$ be a subset of a $\Gamma$-ring $M$. Two right centralizers $T_{1}$ and $T_{2}$ on $M$ are called generalized strong commutativity preserving (GSCP) on $N$ if $\left[T_{1}(a), T_{2}(b)\right]_{\alpha}=[a, b]_{\alpha}$, for all $a, b \in N$ and $\alpha \in \Gamma$. And are called generalized commutativity preserving (GCP) on $N$ if $\left[T_{1}(a), T_{2}(b)\right]_{\alpha}=0$, for all $a, b \in N$ and $\alpha \in \Gamma$.

Example 2.2 Let $M=\left\{\left(\begin{array}{cc}a & b \\ 0 & 0\end{array}\right), a, b \in R\right.$, where $R$ is a ring of intergers $\}$ and $\Gamma=\left\{\left(\begin{array}{cc}n & 0 \\ 0 & m\end{array}\right)\right.$, $n, m \in Z$, where $Z$ is a ring of intergers $\}$. Then $M$ is a $\Gamma$-ring under usual addition and multiplication of matrices. Let $N=\left\{\left(\begin{array}{ll}0 & b \\ 0 & 0\end{array}\right), b \in R\right\}$, then $N$ is a left ideal of $M$. Define mappings $T_{1}$, $T_{2}: M \rightarrow M$ as follows:

$$
T_{1}\left(\begin{array}{ll}
a & b \\
0 & 0
\end{array}\right)=\left(\begin{array}{ll}
a & 0 \\
0 & 0
\end{array}\right) \text { and } T_{2}\left(\begin{array}{cc}
a & b \\
0 & 0
\end{array}\right)=\left(\begin{array}{rr}
0 & -b \\
0 & 0
\end{array}\right) \text {, for all } a, b \in R
$$

Then $T_{1}$ and $T_{2}$ are right centralizers on $M$ and which are GSCP on $N$.

Lemma 2.3. Let $M$ be a semiprime $\Gamma$-ring and $I$ be a non-zero ideal of $M$. If $T$ is a non-zero right centralizer on $M$. Then $T$ is a non-zero on $I$.

Proof. Assume that $T=0$ on $I$ and let $a=a a m$, for all $a \in I, \alpha \in \Gamma, m \in M$.

Then,

It follows that,

$$
0=T(a)=T(a \alpha m)=a \alpha T(m), \text { for all } a \in I, \alpha \in \Gamma, m \in M .
$$

$$
I \Gamma T(M)=0 \text { and } I Г M Г T(M)=\{0\}
$$

Let $\mathrm{P}=\left\{\mathrm{p}_{\mathrm{i}}, \mathrm{i} \in \Lambda\right\}$ be a family of prime ideals of $M$ such that $\cap \mathrm{p}_{\mathrm{i}}=\{0\}$. If $\mathrm{p}_{\mathrm{i}}$ is a typical member of $\mathrm{P}$, then by (1), it follows that

$$
I \Gamma M \Gamma T(M)=\{0\}=\cap \mathrm{p}_{\mathrm{i}} \text { and hence } I \Gamma M \Gamma T(M) \subseteq \mathrm{p}_{\mathrm{i}} \text {, for all } \mathrm{i} \in \Lambda .
$$

By primeness of $\mathrm{p}_{\mathrm{i}}$, we have

Either $I \subseteq \mathrm{p}_{\mathrm{i}}$ or $T(M) \subseteq \mathrm{p}_{\mathrm{i}}$, for all $\mathrm{i} \in \Lambda$.

Using the fact that $\cap \mathrm{p}_{\mathrm{i}}=\{0\}$, we conclude that, Either $I=0$ or $T(M)=0$, 
a contradiction with assumption, then $T$ is a non-zero on $I$.

Theorem 2.4. Let $M$ be a semiprime $\Gamma$-ring of characteristic different from 2 and $N$ be an ideal of $M$. If $T: M \rightarrow M$ is an additive mapping which satisfies $T(a \alpha a)=T(a) \alpha a$, for all $a \in N, \alpha \in \Gamma$, then $M$ contains a central idea.

Proof. By given hypothesis, we have

$$
T(a \alpha a)=T(a) \alpha a, \text { for all } a \in N, \alpha \in \Gamma
$$

Replacing $a$ by $a+b$, where $b \in N$ in (1), we get $T((a+b) a(a+b))=T(a+b) a(a+b)$,

Imply $T((a+b) \alpha a+(a+b) \alpha b)=(T(a)+T(b)) \alpha(a+b)$,

Then $T(a \alpha a)+T(a \alpha b+b \alpha a)+T(b \alpha b)=T(a) \alpha a+T(a) \alpha b+T(b) \alpha a+T(b) \alpha b$,

Using (1), we have $T(a a b+b a a)=T(a) a b+T(b) \alpha a$, for all $a, b \in N, a \in \Gamma$ (2)

Replacing $b$ by $a \beta b+b \beta a$, where $\beta \in \Gamma$ in (2), we get $T(a \alpha(a \beta b+b \beta a)+(a \beta b+b \beta a) \alpha a)=$ $T(a) \alpha(a \beta b+b \beta a)+T(a \beta b+b \beta a) \alpha a$,

Thus, $T(a \alpha(a \beta b+b \beta a)+(a \beta b+b \beta a) \alpha a)=$

$T(a) \alpha(a \beta b)+2 T(a) \alpha(b \beta a)+T(b) \beta(a \alpha a)$, for all $a, b \in N, \alpha, \beta \in \Gamma$

But this can also calculated in a different way,

$T((a \alpha a) \beta b+b \beta(a \alpha a))+2 T(a \alpha(b \beta a))=T(a \alpha a) \beta b+T(b) \beta(a \alpha a)+2 T(a \alpha(b \beta a))$, (by(2)) Using (1), we have $T((a \alpha a) \beta b+b \beta(a \alpha a))+2 T(a \alpha(b \beta a))=(T(a) \alpha a) \beta b+T(b) \beta(a \alpha a)+2 T(a \alpha(b \beta a))$, for all $a$, $b \in N, \alpha, \beta \in \Gamma$

Comparing (3) and (4), we get $T(a \alpha(b \beta a))=T(a) \alpha(b \beta a)$, for all $a, b \in N, \alpha, \beta \in \Gamma$ (5) Replacing $a$ by $a+c$, where $c \in N$ in (5), we have $T((a+c) \alpha(b \beta(a+c))=T(a+c) \alpha(b \beta(a+c))$, Imply $T((a+c) \alpha(b \beta a+b \beta c))=T(a+c) \alpha(b \beta a+b \beta c))$, So

$T(a \alpha(b \beta a)+c \alpha(b \beta a)+a \alpha(b \beta c)+c \alpha(b \beta c))=(T(a)+T(c)) \alpha(b \beta a)+(T(a)+T(c)) \alpha(b \beta c)$

Thus $T(a \alpha(b \beta a))+T(a \alpha(b \beta c)+c \alpha(b \beta a))+T(c \alpha(b \beta c))=T(a) \alpha(b \beta a)+T(a) \alpha(b \beta c)+T(c) \alpha(b \beta a)$ $+T(c)) \alpha(b \beta c)$, for all $a, b, c \in N, \alpha, \beta \in \Gamma$.

Using (5), we get $T(a \alpha(b \beta c)+c \alpha(b \beta a))=T(a) \alpha(b \beta c)+T(c) \alpha(b \beta a)$, for all $a, b, c \in N, \alpha, \beta \in \Gamma$. (6)

Now, we shall compute

$\mathrm{J}=T(a a b \beta c \gamma b \alpha a+b \alpha a \beta c \gamma a \alpha b)$ in two different ways, where $\gamma \in \Gamma$. Using (5), we have

$\mathrm{J}=T(a \alpha((b \beta c \gamma b) \alpha a)+T(b a((a \beta c \gamma a) \alpha b)=T(a) \alpha(b \beta c \gamma b) \alpha a)+T(b) \alpha((a \beta c \gamma a) \alpha b)$,

And using (6), we get

$\mathrm{J}=T((a \alpha b) \beta c \mathcal{\gamma}(b \alpha a)+(b \alpha a) \beta c \mathcal{\gamma}(a \alpha b))=T(a \alpha b) \beta c \mathcal{\gamma}(b \alpha a)+T(b \alpha a) \beta c \mathcal{\gamma}(a \alpha b)$,
Comparing (7) and (8), we have $T(a \alpha b) \beta(c \mathcal{\gamma}(b \alpha a))-(T(a) \alpha b) \beta(c \mathcal{\gamma}(b \alpha a))+T(b \alpha a) \beta(c \mathcal{\gamma}(a \alpha b))-$ $(T(b) \alpha a) \beta(c \gamma(a \alpha b))=0$,

Hence

$(T(a \alpha b)-T(a) \alpha b) \beta(c \mathcal{\gamma}(b \alpha a))+(T(b \alpha a)-T(b) \alpha a) \beta(c \mathcal{h}(a \alpha b))=0$, for all $a, b, c \in N, \alpha, \beta, \gamma \in \Gamma$ (9)

Equation (2) can be written as $T(a \alpha b)-T(a) \alpha b=-(T(b \alpha a)-T(b) \alpha a)$, for all $a, b \in N, \alpha \in \Gamma$.

Using this relation in (9), we get

$$
(T(a \alpha b)-T(a) \alpha b) \beta c) \gamma[b, a]_{\alpha}=0 \text {, for all } a, b, c \in N, \alpha, \beta, \gamma \in \Gamma \text {. }
$$

Using Lemma 1.2, we have

$(T(a a b)-T(a) \alpha b) \beta c) \gamma[u, v]_{\alpha}=0$, for all $a, b, c, u, v \in N, \alpha, \beta, \gamma \in \Gamma$.

Now fix some $a, b$ in $N$ and write $B$ instead of $T(a a b)-T(a) a b)$, we get

$B \Gamma c \Gamma[u, v]_{\alpha}=0$, for all $c, u, v \in N, \alpha \in \Gamma$.

Applying Lemma 1.1, we have

$$
B \Gamma u, v]_{\alpha}=0, \text { for all } u, v \in N, \alpha \in \Gamma .
$$

And by using Lemma 1.3, we get There exists an ideal $I$ of $M$ such that $B \in I \subseteq Z(M)$.

Theorem 2.5. Let $M$ be a prime $\Gamma$-ring of characteristic different from 2 and $I$ a non-zero ideal of $M$. If $T_{1}$ and $T_{2}$ be two non-zero right centralizers on $M$ such that $T_{1}$ and $T_{2}$ are GCP on $I$. Then $M$ contains a non-zero central ideal.

Proof. By the given hypothesis, we have

$\left[T_{1}(a), T_{2}(b)\right]_{\alpha}=0$, for all $a, b \in I$ and $\alpha \in \Gamma$.

Replacing $a$ by $b \beta a$, where $\beta \in \Gamma$ in (1), we get

$$
0=\left[T_{1}(b \beta a), T_{2}(b)\right]_{\alpha}=\left[b \beta T_{1}(a), T_{2}(b)\right]_{\alpha}
$$


Thus

$$
=\left[b, T_{2}(b)\right]_{\alpha} \beta T_{l}(a)+b \beta\left[T_{l}(a), T_{2}(b)=\left[b, T_{2}(b)\right]_{\alpha} \beta T_{l}(a) \text {, by }(1)\right. \text {. }
$$

$\left[b, T_{2}(b)\right]_{\alpha} \beta T_{l}(a)=0$, for all $a, b \in I$ and $\alpha, \beta \in \Gamma$.

Replacing $a$ by $c \gamma a$, where $c \in I, \gamma \in \Gamma$ in (2), we have

$$
0=\left[b, T_{2}(b)\right]_{\alpha} \beta T_{l}(c \gamma a)
$$

$=\left[b, T_{2}(b)\right]_{\alpha} \beta c \gamma T_{l}(a)$, for all $a, b, c \in I$ and $\alpha, \beta, \gamma \in \Gamma$.

Then

And hence,

$$
\left[b, T_{2}(b)\right]_{\alpha} \Gamma I \Gamma T_{I}(a)=0, \text { for all } a, b \in I \text { and } \alpha \in \Gamma .
$$

$\left[b, T_{2}(b)\right]_{\alpha} \Gamma I \Gamma M \Gamma T_{l}(a)=\{0\}$, for all $a, b \in I$ and $\alpha \in \Gamma$.

Since $M$ is a semiprime, then it must contain a family $\mathrm{P}=\left\{\mathrm{p}_{\mathrm{i}}, \mathrm{i} \in \Lambda\right\}$ of prime ideals of $M$ such that $\cap$ $\mathrm{p}_{\mathrm{i}}=\{0\}$. If $\mathrm{p}_{\mathrm{i}}$ is a typical member of $\mathrm{P}$, then by (4), it follows that

$\Lambda$.

$\left[b, T_{2}(b)\right]_{\alpha} \Gamma I \Gamma M \Gamma T_{l}(a)=\{0\}=\cap \mathrm{p}_{\mathrm{i}}$ and hence $\left[b, T_{2}(b)\right]_{\alpha} \Gamma I \Gamma M \Gamma T_{l}(a) \subseteq \mathrm{p}_{\mathrm{i}}$, for all $\mathrm{i} \in$

By primeness of $\mathrm{p}_{\mathrm{i}}$, we have

Either $T_{I}(a) \in \mathrm{p}_{\mathrm{i}}$ or $\left[b, T_{2}(b)\right]_{\alpha} \Gamma I \subseteq \mathrm{p}_{\mathrm{i}}$, for all $a, b \in I$ and $\alpha \in \Gamma, \mathrm{i} \in \Lambda$.

Now, using the fact that $\cap p_{\mathrm{i}}=\{0\}$, we get

Either $T_{1}(a)=0$ or $\left[b, T_{2}(b)\right]_{\alpha} \Gamma I=0$, for all $a, b \in I$ and $\alpha \in \Gamma$.

Since $T_{l}$ is non-zero on $M$, then by Lemma 2.3, we have $T_{l}$ is non-zero on $I$.

Thus

$\left[b, T_{2}(b)\right]_{\alpha} \Gamma I=0$, for all $b \in I$ and $\alpha \in \Gamma$, and hence $\left[b, T_{2}(b)\right]_{\alpha} \Gamma M \Gamma I=\{0\}=\cap \mathrm{p}_{\mathrm{i}}$, for all $b \in I$ and $\alpha \in \Gamma$.

Then, $\left[b, T_{2}(b)\right]_{\alpha} \Gamma M \Gamma I \subseteq \mathrm{p}_{\mathrm{i}}$, for all $b \in I$ and $\alpha \in \Gamma$, i $\in \Lambda$.

So,

Either $I \subseteq \mathrm{p}_{\mathrm{i}}$ or $\left[b, T_{2}(b)\right]_{\alpha} \in \mathrm{p}_{\mathrm{i}}$, for all $b \in I$ and $\alpha \in \Gamma$, i $\in \Lambda$.

Using the fact that $\cap p_{\mathrm{i}}=\{0\}$, we conclude that,

Either $I=0$ or $\left[b, T_{2}(b)\right]_{\alpha}=0$, for all $b \in I$ and $\alpha \in \Gamma$.

Since $I$ is a non-zero ideal, then $\left[b, T_{2}(b)\right]_{\alpha}=0$, for all $b \in I$ and $\alpha \in \Gamma$.

Therefore,

Hence

$T_{2}(b a b)=b \alpha T_{2}(b)=T_{2}(b) \alpha b$, for all $b \in I$ and $\alpha \in \Gamma$, by $T_{2}$ is right centralizer.

$T_{2}(b a b)=T_{2}(b) \alpha b$, for all $b \in I$ and $\alpha \in \Gamma$.

Therefore, $M$ contains a non-zero central ideal by Theorem 2.4.

Corollary 2.6. Let $M$ be a prime $\Gamma$-ring of characteristic different from 2 and $I$ a non-zero ideal of $M$. If $T_{1}$ and $T_{2}$ be two non-zero right centralizers on $M$ such that $T_{1}$ and $T_{2}$ are GCP on $I$. Then $M$ is a commutative $\Gamma$-ring.

Theorem 2.7. Let $M$ be a semiprime $\Gamma$-ring of characteristic different from 2 and $I$ a non-zero ideal of $M$. If $T_{1}$ and $T_{2}$ be two non-zero right centralizers on $M$ such that $T_{1}$ and $T_{2}$ are GSCP on $I$. Then $M$ contains a non-zero central ideal.

Proof. By the given hypothesis, we have

$$
\left[T_{1}(a), T_{2}(b)\right]_{\alpha}=[a, b]_{\alpha} \text {, for all } a, b \in I \text { and } \alpha \in \Gamma \text {. }
$$

Replacing $a$ by $b \beta a$, where $\beta \in \Gamma$ in (1), we get $\left[T_{1}(b \beta a), T_{2}(b)\right]_{\alpha}=[b \beta a, b]_{\alpha}$, for all $a, b \in I$ and $\alpha, \beta$ $\in \Gamma$.

Then $\left[b \beta T_{1}(a), T_{2}(b)\right]_{\alpha}=[b \beta a, b]_{\alpha}$, for all $a, b \in I$ and $\alpha, \beta \in \Gamma$.

Thus,

Using (1), we have

$$
\left[b, T_{2}(b)\right]_{\alpha} \beta T_{l}(a)+b \beta\left[T_{l}(a), T_{2}(b)\right]_{\alpha}=[b, b]_{\alpha} \beta a+b \beta[a, b]_{\alpha}
$$

$$
\left[b, T_{2}(b)\right]_{\alpha} \beta T_{l}(a)=0 \text {, for all } a, b \in I \text { and } \alpha, \beta \in \Gamma \text {. }
$$

By the same method of proof in Theorem 2.5, we complete the proof.

Corollary 2.9. Let $M$ be a prime $\Gamma$-ring of characteristic different from 2 and $I$ a non-zero ideal of $M$. If $T_{1}$ and $T_{2}$ be two non-zero right centralizers on $M$ such that $T_{1}$ and $T_{2}$ are GSCP on $I$. Then $M$ is a commutative $\Gamma$-ring. 


\section{Generalized strong Cocommutativity preserving centralizers on semiprime $\Gamma$-ring.}

We introduce the following definition.

Definition 3.1. Let $N$ be a subset of a $\Gamma$-ring $M$. Two right centralizers $T_{1}$ and $T_{2}$ on $M$ are said to be generalized strong Cocommutativity preserving (GSCCP) on $N$ if $\left(T_{1}(a){ }_{0} T_{2}(b)\right)_{\alpha}=(a \mathrm{o} b)_{\alpha}$, for all $a, b \in$ $N$ and $\alpha \in \Gamma$. And are called generalized Cocommutativity preserving (GCCP) on $N$ if $\left(T_{1}(a) \mathrm{o} T_{2}(b)\right)_{\alpha}$ $=0$, for all $a, b \in N$ and $\alpha \in \Gamma$.

We shall make use of the following commutator identities by condition(A):

$(a \mathrm{o}(b \beta c))_{\alpha}=(a \mathrm{o} b)_{\alpha} \beta c-b \beta[a, c]_{\alpha}=b \beta(a \mathrm{oo} c)_{\alpha}+[a, b]_{\alpha} \beta c$.

$((a \beta b) \mathrm{oc})_{\alpha}=a \beta(b \mathrm{oc})_{\alpha}-[a, c]_{\alpha} \beta c=(a \mathrm{oc})_{\alpha} b \beta+a \beta[b, c]_{\alpha}$.

Theorem 3.2. Let $M$ be a semiprime $\Gamma$-ring of characteristic different from 2, $I$ a non-zero ideal of $M$ and $T_{1}, T_{2}$ be two non-zero right centralizers on $M$ such that $T_{1}$ and $T_{2}$ are GCCP on $I$. Then $M$ contains a non-zero central ideal.

Proof. By the given hypothesis, we have

$$
\left(T_{1}(a) \mathrm{o} T_{2}(b)\right)_{\alpha}=0 \text {, for all } a, b \in I \text { and } \alpha \in \Gamma \text {. }
$$

Replacing $b$ by $a \beta b$, where $\beta \in \Gamma$ in (1), we get

$$
0=\left(T_{1}(a) \mathrm{o} T_{2}(a \beta b)\right)_{\alpha}=\left(T_{l}(a) \mathrm{o}\left(a \beta T_{2}(b)\right)\right)_{\alpha} \text {, for all } a, b \in I \text { and } \alpha, \beta \in \Gamma \text {. }
$$

We have $a \beta\left(T_{I}(a) \mathrm{o} T_{2}(b)\right)_{\alpha}+\left[T_{I}(a), a\right]_{\alpha} \beta T_{2}(b)=0$, for all $a, b \in I$ and $\alpha, \beta \in \Gamma$.

by using (1), we get

$$
\left[T_{I}(a), a\right]_{\alpha} \beta T_{2}(b)=0 \text {, for all } a, b \in I \text { and } \alpha, \beta \in \Gamma \text {. }
$$

Replacing $b$ by $c \gamma b$, where $c \in I, \gamma \in \Gamma$ in (2), we have

$$
\begin{aligned}
0 & =\left[T_{1}(a), a\right]_{\alpha} \beta T_{2}(c \gamma b) \\
& =\left[T_{I}(a), a\right]_{\alpha} \beta c \gamma T_{2}(b), \text { for all } a, b, c \in I \text { and } \alpha, \beta, \gamma \in \Gamma .
\end{aligned}
$$

Then $\quad\left[T_{I}(a), a\right]_{\alpha} \Gamma I \Gamma T_{2}(b)=0$, for all $a, b \in I$ and $\alpha \in \Gamma$.

And hence,

$\left[T_{I}(a), a\right]_{\alpha} \Gamma I \Gamma M \Gamma T_{2}(b)=\{0\}$, for all $a, b \in I$ and $\alpha \in \Gamma$.

Since $M$ is a semiprime, then it must contain a family $\mathrm{P}=\left\{\mathrm{p}_{\mathrm{i}}, \mathrm{i} \in \Lambda\right\}$ of prime ideals of $M$ such that $\cap$ $\mathrm{p}_{\mathrm{i}}=\{0\}$. If $\mathrm{p}_{\mathrm{i}}$ is a typical member of $\mathrm{P}$, then by (4), it follows that

$\left[T_{l}(a), a\right]_{\alpha} \Gamma I \Gamma M \Gamma T_{2}(b)=\{0\}=\cap \mathrm{p}_{\mathrm{i}}$ and hence $\left[T_{l}(a), a\right]_{\alpha} \Gamma I \Gamma M \Gamma T_{2}(b) \subseteq \mathrm{p}_{\mathrm{i}}$, for all $\mathrm{i} \in \Lambda$.

By primeness of $\mathrm{p}_{\mathrm{i}}$, we have

Either $T_{2}(b) \in \mathrm{p}_{\mathrm{i}}$ or $\left[T_{l}(a), a\right]_{\alpha} \Gamma I \subseteq \mathrm{p}_{\mathrm{i}}$, for all $a, b \in I$ and $\alpha \in \Gamma, \mathrm{i} \in \Lambda$.

Using the fact that $\cap \mathrm{p}_{\mathrm{i}}=\{0\}$, we conclude that,

Either $T_{2}(b)=0$ or $\left[T_{1}(a), a\right]_{\alpha} \Gamma I=0$, for all $a, b \in I$ and $\alpha \in \Gamma$.

Since $T_{2}$ is non-zero on $M$, then by Lemma 2.3, we have $T_{2}$ is non-zero on $I$.

Thus

$\left[T_{I}(a), a\right]_{\alpha} \Gamma I=0$, for all $a \in I$ and $\alpha \in \Gamma$, and hence

$\left[T_{I}(a), a\right]_{\alpha} \Gamma M \Gamma I=\{0\}=\cap \mathrm{p}_{\mathrm{i}}$, for all $a \in I$ and $\alpha \in \Gamma$.

Then,

$\left[T_{I}(a), a\right]_{\alpha} \Gamma M \Gamma I \subseteq \mathrm{p}_{\mathrm{i}}$, for all $a \in I$ and $\alpha \in \Gamma, \mathrm{i} \in \Lambda$.

So,

Either $I \subseteq \mathrm{p}_{\mathrm{i}}$ or $\left[T_{l}(a), a\right]_{\alpha} \in \mathrm{p}_{\mathrm{i}}$, for all $a \in I$ and $\alpha \in \Gamma, \mathrm{i} \in \Lambda$.

Using the fact that $\cap p_{\mathrm{i}}=\{0\}$, we have,

Either $I=0$ or $\left[T_{I}(a), a\right]_{\alpha}=0$, for all $a \in I$ and $\alpha \in \Gamma$.

Since $I$ is a non-zero ideal, then $\left[T_{I}(a), a\right]_{\alpha}=0$, for all $a \in I$ and $\alpha \in \Gamma$.

Therefore,

Hence

$$
T_{l}(a) \alpha a=a \alpha T_{l}(a)=T_{1}(a \alpha a) \text {, for all } a \in I \text { and } \alpha \in \Gamma .
$$

$T_{1}(a \alpha a)=T_{1}(a) \alpha a$, for all $a \in I$ and $\alpha \in \Gamma$.

Therefore, $M$ contains a non-zero central ideal by Theorem 2.4.

Corollary 3.3. Let $M$ be a prime $\Gamma$-ring of characteristic different from $2, I$ a non-zero ideal of $M$ and $T_{1}, T_{2}$ be two non-zero right centralizers on $M$ such that $T_{1}$ and $T_{2}$ are GCCP on $I$. Then $M$ is a commutative $\Gamma$-ring.

Theorem 3.4. Let $M$ be a semiprime $\Gamma$-ring of characteristic different from 2, $I$ a non-zero ideal of $M$ and $T_{1}, T_{2}$ be two non-zero right centralizers on $M$ such that $T_{1}$ and $T_{2}$ are GSCCP on $I$. Then $M$ contains a non-zero central ideal. 
Proof. By the given hypothesis, we have

$\left(T_{1}(a) \mathrm{o} T_{2}(b)\right)_{\alpha}=(a \mathrm{o} b)_{\alpha}$, for all $a, b \in I$ and $\alpha \in \Gamma$.

Replacing $b$ by $a \beta b$, where $\beta \in \Gamma$ in (1), we get

Imply,

$\left(T_{1}(a) \mathrm{o} T_{2}(a \beta b)\right)_{\alpha}=(a \mathrm{o}(a \beta b))_{\alpha}$, for all $a, b \in I$ and $\alpha, \beta \in \Gamma$.

Then,

$$
\left(T_{1}(a) \mathrm{o}\left(a \beta T_{2}(b)\right)\right)_{\alpha}=(a \mathrm{o}(a \beta b))_{\alpha} \text {, for all } a, b \in I \text { and } \alpha, \beta \in \Gamma \text {. }
$$

Using (1), we get

$$
a \beta\left(T_{l}(a) \mathrm{o} T_{2}(b)\right)_{\alpha}+\left[T_{I}(a), a\right]_{\alpha} \beta T_{2}(b)=a \beta(a \mathrm{o} b)_{\alpha}+[a, a]_{\alpha} \beta b
$$

$\left[T_{I}(a), a\right]_{\alpha} \beta T_{2}(b)=0$, for all $a, b \in I$ and $\alpha, \beta \in \Gamma$.

By the same method of proof in Theorem 3.2, we complete the proof.

Corollary 3.5. Let $M$ be a prime $\Gamma$-ring of characteristic different from $2, I$ a non-zero ideal of $M$ and $T_{1}, T_{2}$ be two non-zero right centralizers on $M$ such that $T_{1}$ and $T_{2}$ are GSCCP on $I$. Then $M$ is a commutative $\Gamma$-ring.

Now, by using similar techniques as in the Theorems 2.7 and 3.4, we get the following result.

Theorem 3.6. Let $M$ be a semiprime $\Gamma$-ring of characteristic different from $2, I$ a non-zero ideal of $M$ and $T_{1}, T_{2}$ be two non-zero right centralizers on $M$. Then $M$ contains a non-zero central ideal, if one of the following conditions holds:

(i) $\quad\left[T_{1}(a), T_{2}(b)\right]_{\alpha}+[a, b]_{\alpha}=0$, for all $a, b \in I$ and $\alpha \in \Gamma$.

(ii) $\quad\left(T_{1}(a) \mathrm{o}_{2}(b)\right)_{\alpha}+(a \mathrm{o} b)_{\alpha}=0$, for all $a, b \in I$ and $\alpha \in \Gamma$.

(iii) $\quad\left[T_{1}(a), T_{2}(b)\right]_{\alpha}=(a \mathrm{o} b)_{\alpha}$, for all $a, b \in I$ and $\alpha \in \Gamma$.

(iv) $\quad\left[T_{1}(a), T_{2}(b)\right]_{\alpha}+(a \mathrm{o} b)_{\alpha}=0$, for all $a, b \in I$ and $\alpha \in \Gamma$.

\section{References}

1. Nobusawa, N. 1964. On a generalization of the ring theory, Osaka J. Math., (1): 81-89.

2. Barnes, W. E. 1966. On the $\Gamma$-rings of Nobusawa, Pacific J. Math. 18(3): 411-422.

3. Kyuno, S. 1978. On prime gamma ring, Pacific J. Math.75: 185-190.

4. Luh, J. 1969. On the theory of simple $\Gamma$-rings, Michigan Math. J., 16(1): 65-75.

5. Zalar, B. 1991. On centralizers of semiprime rings, Comment. Math. Univ. Carolinae, 32: 609614.

6. Vukman, J. 1997. Centralizers in prime and semiprime rings, Comment. Math. Univ. Carolinae, 38: $231-240$.

7. Vukman, J. 1999. An identity related to centralizers in semiprime rings, Comment. Math. Univ. Carolinae, 40(3): 447-456.

8. Vukman, J. 2001. Centralizers on semiprime rings, Comment. Math. Univ. Carolinae, 42(2): 237245.

9. Hoque, M. F. and Paul, A. C. 2011. On centralizers of semiprime gamma rings, International Mathematical Forum, 6(13): 627- 638.

10. Hoque, M. F. and Paul, A. C. 2013. Centralizers of semiprime gamma rings, Italian J. Pure and Applied Mathematics, 30: 289- 302.

11. Hoque, M. F. and Paul, A. C. 2012. An equation related to centralizers in semiprime gamma rings, Annals of Pure and Applied Mathematics, 1(1): 84- 90.

12. Haker, H. A. 2005. Centralizers on prime and semiprime rings, Department of Mathematics, University of Baghdad, Baghdad, Iraq.

13. Abduljaleel, A. A. 2017. Commutativity results on prime and semiprime rings, M.Sc. Thesis. Department of Mathematics, University of Baghdad, Baghdad, Iraq. 